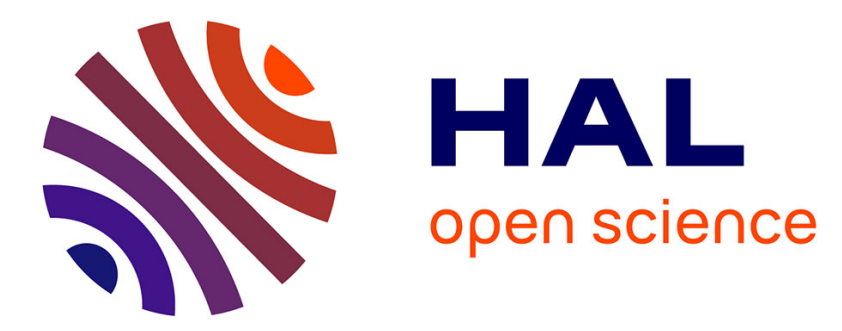

\title{
Relecture par un écologue des principaux écrits sur la restauration d'espaces érodés dans les Alpes du sud (1797-1994).
}

\author{
Daniel Vallauri
}

\section{- To cite this version:}

Daniel Vallauri. Relecture par un écologue des principaux écrits sur la restauration d'espaces érodés dans les Alpes du sud (1797-1994).. Revue forestière française, 1998, 50 (4), pp.367-378. $10.4267 / 2042 / 5550$. hal-03443535

\section{HAL Id: hal-03443535 \\ https://hal.science/hal-03443535}

Submitted on 23 Nov 2021

HAL is a multi-disciplinary open access archive for the deposit and dissemination of scientific research documents, whether they are published or not. The documents may come from teaching and research institutions in France or abroad, or from public or private research centers.
L'archive ouverte pluridisciplinaire HAL, est destinée au dépôt et à la diffusion de documents scientifiques de niveau recherche, publiés ou non, émanant des établissements d'enseignement et de recherche français ou étrangers, des laboratoires publics ou privés. 


\section{nature, histoire loisirs et forêt}

\section{RELECTURE PAR UN ÉCOLOGUE DES PRINCIPAUX ÉCRITS SUR LA RESTAURATION D'ESPACES ÉRODÉS DANS LES ALPES DU SUD (1797-1994)}

\section{VALLAURI}

Conséquence de la réflexion engagée sur le développement durable, les besoins de restauration d'espaces dégradés réapparaissent comme une préoccupation moderne dans l'aménagement des territoires. Une discipline de l'écologie, l'écologie de la restauration, se structure actuellement autour de ce thème et développe des recherches tant fondamentales que finalisées. En France, le programme national de recherche "Recréer la nature", lancé par le ministère de l'Environnement et le Muséum national d'Histoire naturelle depuis 1997, se propose d'affiner dans ce sens les approches techniques, écologiques, voire socio-économiques, nécessaires à la mise en place d'un projet de restauration.

Dans les Alpes, grâce à la politique de Restauration des Terrains en Montagne (RTM), une expérience s'est forgée par le biais de programmes centenaires visant à une lutte active contre les phénomènes redoutés (érosion, crues torrentielles, avalanches). Parmi ceux-ci, celui consistant à lutter contre l'érosion dans les Alpes du Sud est un exemple particulièrement enrichissant pour :

- aborder les problèmes similaires et actuels, dans les montagnes méditerranéennes notamment ;

- tirer les enseignements d'un siècle d'application de techniques de réhabilitation ;

- répondre aux interrogations écologiques et techniques des gestionnaires forestiers sudalpins (viabilité des peuplements créés, nature de l'accompagnement sylvicole).

Aussi, nous a-t-il paru utile de redonner la parole aux principaux acteurs scientifiques et techniques de cette "entreprise centenaire" (de Crécy, 1982), afin de mettre en perspective l'évolution des idées 


\section{VALLAURI}

concernant la restauration. Réalisée à partir de citations, sélectionnées pour illustrer le questionnement actuel de l'écologue de la restauration, cette compilation rappelle très clairement certaines idées de base, parfois fort anciennes: la recherche de référence et d'objectif à long terme, le phasage de la restauration, la stratégie pour les travaux, le choix des végétaux,...

Nous présentons les contributions par grande phase historique, débutant ainsi par les scientifiques ayant attiré l'attention sur le problème dès la fin du XVIII e siècle, poursuivant par les maîtres d'œuvre de la restauration dans les Alpes du Sud durant le $\mathrm{XIX}^{\mathrm{e}}$ siècle et, enfin, terminant par les acteurs forestiers contemporains. Le déroulement du programme de restauration est décomposé en trois actes, suivant ainsi le processus de restauration des écosystèmes forestiers (Vallauri et Chauvin, 1997 ; Vallauri et al., 1997) :

- l'acte 1 présente la longue phase de prise de conscience du caractère non naturel de l'érosion généralisée des bassins versants et la nécessité de restaurer la végétation;

- l'acte 2 est relatif à la mise en œuvre de la restauration. Si l'important travail législatif et d'organisation administrative des programmes de restauration n'est pas analysé ici, nous nous attardons par contre sur les concepts et techniques développés lors de la phase de réhabilitation des terrains (génie civil, reboisement et/ou revégétalisation des espaces érodés) ;

- l'acte 3 correspond aux nécessaires travaux d'accompagnement des écosystèmes réhabilités (suivi et pilotage).

Enfin, l'épilogue est ébauché en conclusion et consiste en la définition de règles pour une gestion durable de ces nouvelles forêts à rôle prioritaire de protection.

\section{ACTE 1. PHASE DE PRISE DE CONSCIENCE (1797-1860)}

Quatre textes ont plus particulièrement conduit à une prise de conscience du besoin de restauration de la végétation pour lutter contre l'érosion, et cela à des titres divers :

1. en lançant le débat sur les causes anthropiques de la dégradation des territoires, par le déboisement de substrats sensibles (Fabre, 1797 ; Rauch, 1818). Fabre (1797) présente, dans un ouvrage dédié à l'hydrologie, l'une des toutes premières mises en cause de la déforestation dans la genèse de nouvelles zones d'érosion et l'augmentation du charriage des torrents. Quant à l'ouvrage de Rauch (1818), au titre éloquent, il fait suite à un ouvrage du même auteur intitulé "Harmonie hydro-végétale et météorologique" (1802) et présente le premier exposé raisonné sur les conséquences du déboisement ;

2. en présentant, au-delà du simple arrêt des dégradations et compte tenu de leur importance, l'action de restauration comme indispensable (Rauch, 1818). Rauch introduit le terme de "régénération de la nature végétale", correspondant à l'acception biologique du terme de "restauration", qui n'apparaît que dans les années 1860, et est équivalent de l'actuelle "restauration écologique". Cependant, il s'agit pour lui plus de présenter des intentions généreuses qu'un véritable projet technique ;

3. en proposant un projet volontaire, complet, argumenté et concret pour les Basses-Alpes (Dugied, 1819). Préfet à Digne pendant huit mois seulement, Dugied se passionne pour la lutte contre la désertification des terres et des hommes. II pose (propose) la première pierre d'une politique ambitieuse et volontaire de restauration du territoire, sous forme d'un projet très élaboré du point de vue des techniques et de l'organisation humaine et financière. En avance sur les idées de son époque, mal appuyé par les connaissances scientifiques sur la faisabilité de la restauration et en opposition d'intérêts avec les acteurs socio-économiques régionaux, ce projet attendra 41 ans avant d'aboutir ; 
4. en démontrant, avec les données de la science de l'époque, que la réhabilitation des terrains érodés était nécessaire et possible dans les Alpes du Sud (Surrel, 1841). Ce dernier ouvrage, couronné par l'Académie des Sciences en 1842, présente une démonstration du processus de formation des torrents à partir de l'observation raisonnée des torrents des Hautes-Alpes. II donne une base scientifique solide (hydrologie torrentielle) au futur programme de restauration, mettant au premier plan sa composante biologique, d'une part pour prévenir la formation de nouveaux torrents, mais surtout pour limiter les torrents déclarés les plus dangereux.

\section{Les causes de la dégradation des terrains en montagnes}

"La destruction des bois qui couvrent nos montagnes est la première cause de la formation des torrents » affirme Fabre dès 1797. Rauch (1818) met également clairement les activités humaines à l'index : "les lois harmoniques de la nature (...) sont interverties par de longs siècles de mutilation ".

II est vrai par exemple que le département des Basses-Alpes "offre le spectacle de la plus triste infertilité " poursuit Dugied (1819). II présente 430613 ha de terrains peu ou improductifs, environ la moitié de sa surface. Ainsi, "en apercevant pour la première fois ces vastes lits de cailloux (Dugied parle du lit des rivières), on se demande quelle puissance inconnue a pu y amener tant de débris; mais lorsqu'on s'élève sur les hauts sommets, que l'œil, après avoir embrassé les monts moins élevés, pénètre jusqu'au fond des vallées, alors le voile qui couvre la cause de tant de ravages se soulève, et l'on reconnaît que l'homme est le principal auteur de la désolation qui règne autour de lui ».

Surrel (1841) observe et décrit rigoureusement les phénomènes : "pour qu'un affouillement se manifeste, il faut qu'une grande force d'érosion agisse sur un terrain susceptible d'être corrodé ". Trois causes principales sont invoquées: sensibilité géologique, météorologie et déboisement. Surrel précise qu'en général, ce n'est " pas la destruction des forêts, ainsi qu'on le croit communément ici, qui a suffi pour attirer sur ces (...) montagnes le fléau si particulier qui les désole ». "Leur influence est incontestable, mais elle eût été nulle sous un autre ciel et dans d'autres terrains ».

\section{Comment arrêter la dégradation des terrains ?}

"S'il reste encore de la terre végétale sur ces montagnes, le mieux serait de les laisser se boiser en laissant ces terres en friche (...). II n'est pas moins essentiel de pourvoir à la conservation des bois existants (...)». Toutefois, "la nature n'est que plus active lorsqu'elle est aidée par l'industrie humaine. Ainsi, dans le cas où l'on voudrait aider sur certains penchants de montagnes la multiplication des bois, il ne serait souvent pas mal d'y semer, soit des glands, soit des faines de l'espèce d'arbre qu'on présumerait être propre aux localités. (...) II y a des cas où il reste assez peu de terre sur les montagnes (...). On pourrait alors, avec succès, gazonner ce terrain. (...) Ce sont là des moyens de prévenir la formation des torrents sur les montagnes » (Fabre, 1797).

\section{Est-il utopique de vouloir restaurer la végétation?}

L'action de restauration pour arrêter l'érosion dans des secteurs déclarés n'est encore pour Fabre (1797) qu'une piste de réflexion à développer à l'avenir. "Il nous reste à voir ce qu'il faut employer pour détruire, lorsque la chose est possible, les torrents déjà formés » conclut-il.

Rauch (1818) poursuit plus loin la réflexion. "Puisque le temps est si lent à reproduire ce que l'homme a détruit dans un instant; puisque des siècles d'imprévoyance ont accumulé sur nous tant de maux qui tiennent au désordre des météores, à l'altération des climatures et à la diminution de toutes les productions de la terre; soyons dociles à la voix du malheur qui nous crie de replanter avec célérité nos antiques montagnes". "La nature, flétrie dans ce qu'elle avoit reçu de plus gracieux de la création, demande à être régénérée ". L'idée de la restauration écologique de nos montagnes est née. 


\section{VALLAURI}

Dans la conclusion de son ouvrage, Dugied (1819) s'engage une fois de plus à titre personnel et précise qu'il est convaincu que "le projet de reboiser les Basses-Alpes, loin d'être une chimère, est parfaitement exécutable ». II imagine le calendrier d'une régénération végétale orchestrée par I'homme : "dix ans de constance, et l'opération me paraissait assurée ; (...) vingt années, et les résultats commençaient; un quart de siècle, ils étaient évidents pour tous; un demi siècle, et la plus belle entreprise que puissent essayer les hommes, celle de changer la nature (...), (de) rendre la fertilité au sol qu'ils ont désolé, était couronnée du plus éclatant succès ».

Surrel conforte par l'observation ces nobles intentions : "partout où il y a des torrents récents, il n'y a plus de forêts, et partout où l'on a déboisé le sol, des torrents récents se sont formés; en sorte que les mêmes yeux qui ont vu tomber les forêts sur le penchant d'une montagne, y ont vu apparaitre incontinent une multitude de torrents ". L'observation et la mémoire des anciens "forcent de reconnaître que les forêts exercent une influence réelle sur la production des torrents ". De plus, "beaucoup de versants boisés portent les traces de petits torrents (...), qui paraissent comme étouffés sous la masse de la végétation, et sont complètement éteints ". Ainsi, "les forêts sont capables de provoquer l'extinction d'un torrent déjà formé ". La restauration est donc loin d'être une utopie pour Surrel (1841), puisque cet "art alors se bornera à imiter la nature, à s'emparer de ses procédés, et à opposer habilement les forces de la vie organique à celle de la matière brute ". Or, "tout ce que nous allons entreprendre la nature l'a déjà fait avant nous dans des temps passés (...). Nous sommes donc assurés d'avance du succès ».

\section{Création ou re-création : quelles références?}

"Il est temps de recréer le passé " (Dugied, 1819). Selon lui, les boisements existaient par le passé, mais si cela n'est pas à l'époque une idée admise par tout le monde, "c'est que tout ce qui concerne les anciennes forêts se perd dans l'obscurité des temps » et que même l'information sur l'histoire récente des forêts est mal connue.

Mais, "le reboisement est-il possible? » (Surrel, 1841). Surrel ne cache pas les difficultés d'installer une forêt sur des terres arides et érodées. Toutefois, "est-ce à dire pour cela qu'il faille se contenter de produire des broussailles (...) ? Non, car les bois donnent de la valeur aux terrains qu'ils recouvrent, ils emportent d'ailleurs avec eux des éléments nouveaux de régénération qui ne se rencontrent pas dans les broussailles. Considérons seulement cette même végétation comme destinée à précéder la grande végétation des forêts, sur les terrains où celle-ci aurait trop de peine à s'installer tout d'abord ».

\section{Est-on prêt pour l'action?}

La restauration écologique de nos montagnes est louée par Rauch (1818) : "ce triomphe du génie de la reproduction sur celui de la destruction et du chaos" écrit-il. Elle est techniquement envisageable. Elle n'est toutefois possible qu'avec un investissement (financier, humain) et un engagement importants de tous les acteurs concernés. Ceux-ci restent à mobiliser et à convaincre. De nombreuses réticences et oppositions subsistent jusqu'au $X X^{\mathbb{e}}$ siècle concernant le bien-fondé, la faisabilité et du fait de l'utilisation des terres par l'agriculture, bien que Rauch affirme dès 1818 que "cette conquête de l'empire de la fécondité, conquête la plus durable et la plus glorieuse, puisqu'elle tend à rétablir, à embellir l'œuvre de Dieu, est aujourd'hui le vœu de tous les Princes et de tous les Gouvernements, elle ne peut que se réaliser pour la gloire et le bonheur de la nature humaine ". Dugied (1819) insiste sur l'application de la législation et son évolution nécessaire, ainsi que sur l'engagement de l'action de l'État pour faire cesser les défrichements en appliquant l'Ordonnance de 1669, pour encourager le semis (don de graines, prime de $20 \mathrm{~F}$ de l'hectare) et pour encaisser les torrents avec une aide financière de $50 \%$ de l'État. Dans les faits, la restauration ne débutera d'ailleurs qu'après une décision politique et la promulgation des lois de 1860 et 1864 . 


\section{Premières réflexions sur les objectifs et les techniques}

Dans le projet de boisement des Basses-Alpes de Dugied (1819), l'objectif principal du reboisement pour lutter contre l'érosion est clair: "c'est dans la vallée que sont et doivent être les terres cultivées ; c'est la vallée qu'il faut protéger ». Surrel ajoute en 1841 que " ce n'est plus un système de défense qu'il faut chercher, mais c'est un double système de préservation et d'extinction ». La préservation (défense passive) nécessite un réseau cohérent de digues et de seuils. Un système d'extinction (défense active) est également proposé et consiste en la délimitation de zones de défense dans le bassin de réception du torrent et en des travaux de reboisement.

Dugied définit les travaux pour la régénération végétale comme "l'ouvrage du temps et de la persévérance ». "Il faut donc semer; que les hommes y mettent la main, qu'ils sachent protéger leur ouvrage, et le temps fera le reste ". II estime à 150000 ha les surfaces présentant "la possibilité de semer avec succès dans le département". II précisera d'ailleurs plus loin, "pour fortifier ses prétentions ", qu' "il s'agit de créer plus de forêts qu'il n'en a existé autrefois dans le département, ou du moins qu'on se le rappelle". Surrel ajoute que "le but de ces travaux doit être de couvrir le bassin de réception par une forêt qui s'épaississe chaque jour, et qui, s'étendant de proche en proche, finisse par l'envahir jusque dans ces derniers replis ».

D'un point de vue technique, Dugied détaille déjà les espèces forestières utilisables en reboisement. Mais il note aussi qu'il pourrait être semé "sur les côtes trop ardues, trop dépouillées de sol végétal, des buis et genêts". Ceux-ci sont une source d'engrais et d'humus pour l'agriculture et "chaque jour il devient plus difficile de s'en procurer, parce que chaque jour il faut aller plus loin pour chercher ces genêts et ces buis ".

\section{ACTE 2. PHASE DE RÉHABILITATION DES TERRAINS (1860-1930)}

À partir de la décision politique de restaurer (se traduisant ici par les lois de 1860 et 1864 et l'engagement de l'État), la première phase des travaux (la réhabilitation) consiste notamment à installer une végétation pionnière. Dans les Alpes du Sud, les techniques ont été affinées au fur et à mesure de leur validation en vraie grandeur dans chaque périmètre de restauration. Aussi trouvet-on dans le document de Mathieu (1865), mais plus encore dans la synthèse réalisée par Demontzey (1878), une somme considérable d'expériences, dont nous n'extrayons ici que quelques aspects.

Professeur à l'École forestière, Mathieu se voit confier la rédaction du premier manuel de reboisement et gazonnement des montagnes, répondant ainsi aux préoccupations de mise en œuvre des lois de 1860 et 1864 . Quant à Demontzey, dont nous célébrons le centième anniversaire de la mort en 1998, nul n'est besoin de le présenter. Son étude se propose de rassembler les enseignements de 16 ans d'expériences en Restauration des Terrains en Montagne. Modestement, Demontzey note que les 421 pages de son ouvrage principal n'ont pas "la prétention de poser des règles générales et absolues ". Cet ouvrage de synthèse donnera pourtant à l'action RTM française un retentissement international. II confortera l'ensemble des programmes conséquents réalisés en France : la surface des périmètres RTM, certes pas restaurés en totalité, est par exemple estimée à 165000 ha dans les seuls départements des Alpes-Maritimes, Hautes-Alpes et Alpes-de-HauteProvence selon Douguedroit (1980).

\section{Objectif et optimisation des travaux}

Mathieu définit tout d'abord comment choisir les périmètres qu'il s'agira de "reboiser, gazonner ou broussailler ". "Loin de tout éteindre (il parle des torrents), il faut donc commencer les efforts sur un petit nombre de périmètres convenablement choisis". "Les forêts doivent être rétablies vers la 


\section{VALLAURI}

base ou la partie moyenne des montagnes, dans les lieux accessibles où leurs produits gagneront en valeur (...). Quant aux pâturages, toutes les fois qu'ils seront destinés aux moutons et aux chèvres, ils pourront sans inconvénient occuper les parties supérieures ». Demontzey (1878) quant à lui insiste sur la nécessité de définir des zones continues de défense suivant les limites hydrologiques du torrent à traiter.

"Il est évident que, dans les conditions où se présentent le plus souvent les terrains destinés au reboisement, on ne peut songer à obtenir indifféremment partout et d'emblée la création d'un peuplement appelé à former la futaie désirée, et que sur bien des points il faudra se contenter d'abord de produire une végétation quelconque et attendre de longues années pour que les conditions de sol se soient suffisamment modifiées»(Demontzey, 1878). Demontzey cite Arthur Young, scientifique de la fin du XVIII ${ }^{e}$ siècle, qui écrivait avec sagesse: "dans de pareils essais, ceux qui les entreprennent veulent toujours aller trop vite au dernier degré de la perfection et rendre tout à coup ces déserts semblables à des champs depuis longtemps cultivés. Pour que de semblables travaux soient au contraire avantageux, il faut éviter les grandes dépenses et laisser quelque chose à faire au temps, qui travaille en silence avec efficacité ».

\section{Les travaux préalables et préparatoires au reboisement}

Préalablement à toute opération de reboisement ou de gazonnement, la correction s'appuie sur le génie civil. "Les travaux de correction doivent avoir le triple but de diminuer la pente du profil en long, de consolider le nouveau lit, et d'élargir sa section dans toutes les parties du torrent susceptibles d'être affouillées ». À cet effet, il doit être prévu un ensemble de zones d'atterrissement, de barrages vivants (clayonnages et fascines) et de barrages successifs en génie civil, tel que "la ligne qui joint le pied du supérieur au couronnement de l'inférieur détermine la pente d'équilibre " (Demontzey, 1878).

Divers travaux préparatoires au reboisement sont également nécessaires, poursuit Demontzey (1878) :

- la mise en défends des terrains à reboiser, pour le raffermissement de la surface du sol et l'extension naturelle de la végétation herbacée. Toutefois, "(...) en ce qui concerne les terrains croulants, glissants, profondément ravinés (...), la mise en défends devient une pure chimère ";

- l'utilisation des "vestiges de végétaux ligneux" avec "recépage complet de tous ces sujets " pour " obtenir une production de rejets abondants et plus vigoureux »;

- «l'enherbement»;

- «le défoncement profond du sol ». Dans les marnes dures, Demontzey préconise d'utiliser la faculté naturelle de la roche à se déliter pendant l'hiver, en préparant, deux ans à l'avance, les potets de plantation.

\section{Le choix des végétaux pour la réhabilitation}

Parmi les espèces à utiliser pour les travaux, Mathieu (1865) "met en première ligne l'emploi des végétaux indigènes ". En effet, "quel meilleur bois désirer que celui de nos chênes, hêtres, frênes, ormes, etc.,... de nos sapins, épicéas, pins, mélèzes; que regretter en eux sinon que la quantité ne corresponde pas à la qualité ? ". II conseille donc de "reboiser avec des espèces éprouvées, c'està-dire, pour le présent, à peu près uniquement avec les essences indigènes ". Toutefois, "la propagation du Pin noir d'Autriche (...) peut être entreprise avec les chances du succès ». Dans un chapitre spécial sur la fixation des terres noires, il précise qu'«il s'agit bien moins de créer des pâturages ou des forêts que de fixer rapidement le sol ». II conseille l'utilisation de matériel végétal drageonnant mais aussi la constitution de seuil en pierres sèches, de fascines mortes ou vivantes. 
Nature, histoire, loisirs et forêt
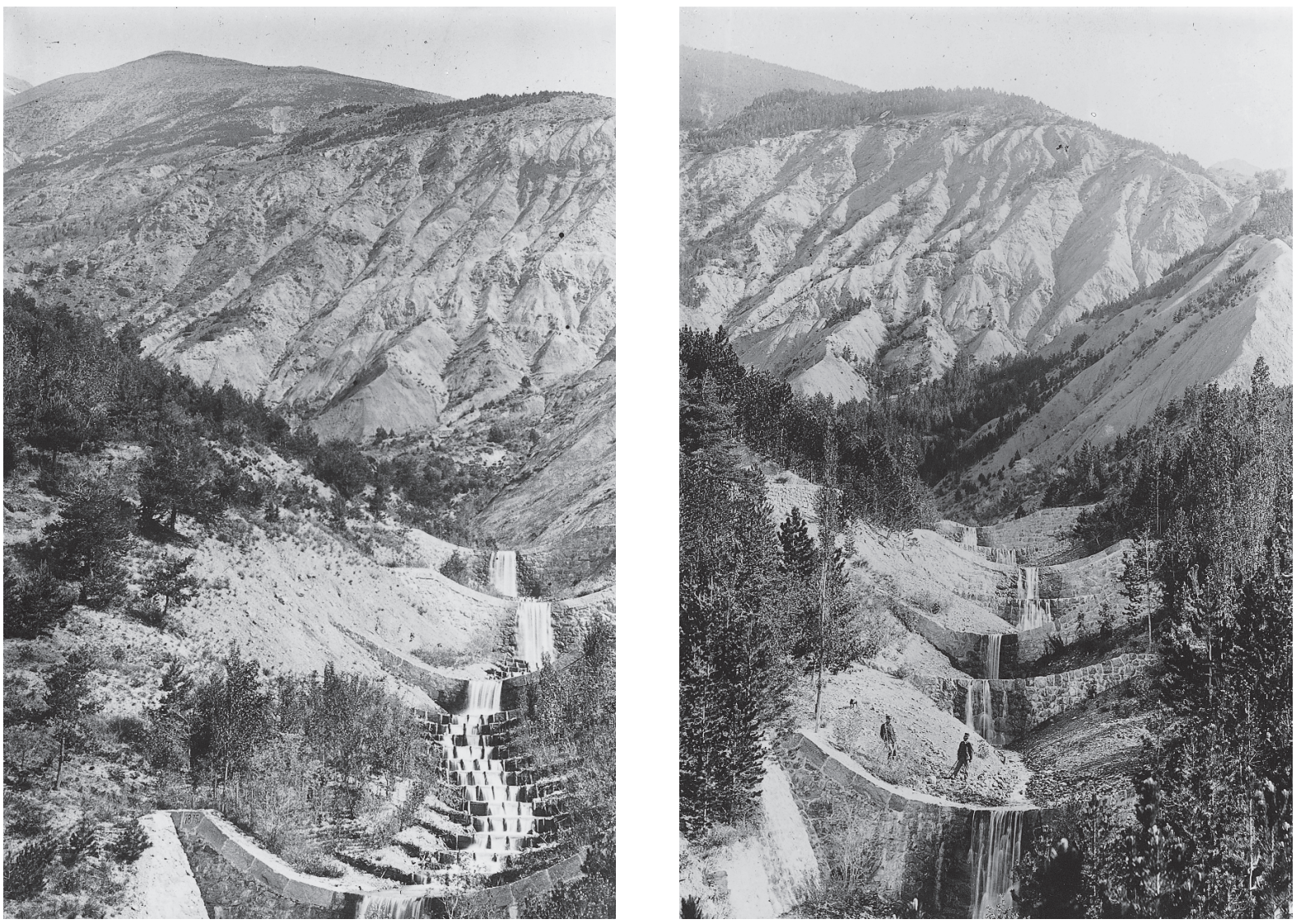

Torrent du Bourget (Savoie), en 1887 (à gauche), en 1905 (à droite)

Photos Collection ENGREF - Nancy

Pour ce qui concerne le reboisement, "le but est la création d'une végétation ligneuse qui réponde aux conditions suivantes: 1. posséder des racines assez puissantes pour enserrer le sol dans leurs innombrables réseaux, le rendre au besoin plus perméable et le protéger contre l'entraînement; 2. présenter un couvert assez complet pour abriter sa surface contre les influences météorologiques ; 3. fournir un humus de plus en plus abondant, appelé d'une part à fertiliser le sol et à augmenter ainsi la puissance de la végétation, et d'autre part à favoriser le ralentissement et la régulation du débit des eaux pluviales; 4. maintenir, sans interruptions momentanées et perpétuellement, ces salutaires effets et les développer avec l'aide du temps » (Demontzey, 1878).

Une longue liste commentée d'espèces à utiliser en reboisement ou en amélioration est présentée par Demontzey pour chaque domaine bioclimatique des Alpes. Elle reprend 9 espèces résineuses, environ 50 espèces feuillues et 9 espèces exotiques (voir la liste reprise dans Vallauri, 1997b). L'enherbement est également envisagé soit en sainfoin, soit en fenasse (brome, boucage, houlque, fromental élevé), soit en bauche (Calamagrostide argentée), soit avec un mélange (3/4 à 4/5 de sainfoin pour $1 / 4$ à $1 / 5$ de graminées). 


\section{VALLAURI}

\section{Les techniques de boisement et de végétalisation}

Reste une question ouverte pour Mathieu (1865): semis ou plant? II rappelle les contraintes extrêmes de ces milieux marneux secs et conclut que "partout où il faut lutter contre le dessèchement du sol, contre la dénudation, contre le déchaussement (...), la plantation bien faite est préférable au semis ». De plus, “ (...) favoriser l'enracinement rapide et profond des jeunes plants, c'est en fortifier le tempérament ». Aussi, préconise-t-il la technique du "pot ouvert de distance en distance (...), dont on ameublit le fond ».

Pour Demontzey, le Pin sylvestre et le Pin noir, fréquemment utilisés sur les substrats marneux, sont deux essences à installer par plantation. En pépinières ou sur le terrain, les semis sont réalisés au printemps pour les résineux et les herbacées. Les résineux produits sont des plants en racines nues qui doivent être plantés immédiatement, si possible sans jauge intermédiaire. Sur les stations médiocres, sur marnes par exemple, les plants ont 3 ans. Ils sont plantés par touffe de 2 à 4 dans un potet, au printemps ou à l'automne. Le nombre de plants selon les techniques varie de 1666 à 10000 à l'hectare.

\section{ACTE 3. PHASE D’ACCOMPAGNEMENT DES ÉCOSYSTÈMES RÉHABILITÉS (1930-2030)}

Comme l'avaient bien évoqué les acteurs de la phase de réhabilitation, la restauration forestière de terrains dégradés par érosion est une entreprise à long terme. Les écologues de la restauration précisent d'ailleurs aujourd'hui qu'il s'agit d'un processus écologique complexe, d'autant plus long que l'écosystème à "recréer" correspond à un stade dynamique plus mature ou diversifié. Par conséquent, cette phase nécessite un suivi technique et un accompagnement sylvicole.

Inspecteur des Eaux et Forêts ayant poursuivi à Digne l'action de Demontzey, Lavauden rédigea au moment de quitter la direction de la conservation (1930) une petite note de 7 pages présentant de nombreuses idées pour poursuivre le processus de restauration. Elle semble malheureusement avoir été suivie de peu d'effets.

Cependant, après quarante ans de mise en veille, les préoccupations techniques sur le devenir des forêts créées ne manquent pas de resurgir dans les années 1970 et 1980. De nombreux auteurs comme Bartet, Combes, Corvol, de Crécy, Douguedroit, Dreyfus, Fourchy, Poncet, Saillet..., se sont attachés, sous l'angle technique ou historique, à retracer l'action de la RTM, à en dresser un bilan et formuler les nouvelles questions posées par les forêts créées.

\section{Un accompagnement de la restauration négligé... des problèmes nouveaux}

"L'œuvre ancienne de reboisement, qu'il faut défendre, entretenir, étendre, pose de nos jours des problèmes concrets, souvent nouveaux, toujours ardus » (de Crécy, 1982). "Le Pin noir a joué son rôle pionnier »; (...) « il fallait un arbre impersonnel (...) pour supporter l'installation dans les landes en érosion et permettre au milieu de se révéler » (Combes et Bartet, 1982). Mais " on a laissé passer l'âge des premières éclaircies, trop coûteuses» (...), et "on s'achemine trop souvent vers le vieillissement massif qui compromettra à la fois l'avenir de la forêt et surtout son rôle anti-érosif » (de Crécy, 1982). D’où "l'urgente nécessité » de mettre au point une véritable " sylviculture des forêts de protection » (de Crécy, 1982).

\section{Diversité et structure de l'écosystème objectif ?}

Lavauden (1930) ne peut être plus clair : " d'abord, et avant tout, il est nécessaire que les forestiers des Basses-Alpes se pénètrent bien de cette notion que les plantations de Pins noirs ne sont jamais un but, et qu'elles ne constituent qu'un moyen. Sans doute elles sont commodes; sans doute elles 
paraissent couvrir le sol, et permettent de croire ou de faire croire à des résultats importants. Mais elles courent des risques énormes (incendie, invasions de processionnaire, etc.). (...) Le but lointain doit être l'établissement d'une forêt (...) voisine des types de forêts qui n'ont point été trop abîmées par l'action humaine ". II note que le Sapin "peut être, dans les Basses-Alpes, beaucoup plus répandu qu'il n'est aujourd'hui » et qu'il devra donc être "planté en sous-étage dans les plantations de Pins noirs éclaircies, ainsi que dans les forêts ou plantations de Pins sylvestres ". D'autre part, "les résineux ne doivent pas être seuls à constituer nos forêts nouvelles. Il faut que celles-ci soient complètes, c'est-à-dire comportent un sous-étage et un sous-bois sans même oublier le tapis herbacé ".

"L'objectif de protection conduit, encore, à recommander (...) la diversité, l'irrégularité des boisements". "Ce n'est pas seulement l'écologie, le souci de l'adaptation du boisement au milieu, mais l'objectif de protection, le souci d'efficacité, qui plaident pour une large part réservée aux feuillus dans les reboisements de protection » (Poncet, 1994).

\section{Intégrer l'hétérogénéité et la dynamique des stations}

"La station, souvent étroitement délimitée, a des aptitudes différentes en matière de végétation et est soumise à des processus érosifs différents". "Cette mosaïque (...) doit être détectée et respectée étroitement par le reboiseur-restaurateur (...) souvent à quelques décamètres ou même quelques mètres près". "L'analyse écologique des stations, recommandée aux aménagistes des forêts pour leur mise en valeur, s'impose donc bien plus étroitement au reboiseur-restaurateur de terrains dégradés » (Poncet, 1994).

Poncet rappelle aussi que "l'évolution naturelle, par stades progressifs de plus en plus riches, du groupement végétal, qui améliore peu à peu son biotope " conforte l'intérêt porté aux espèces pionnières. Toutefois, le "climax" et la forêt "réalisent à la fois la plus forte biomasse et la plus puissante pédogenèse". Ainsi, si "théoriquement les premières plantes à installer seront celles correspondant au stade de dégradation atteint ou dépassé ", (...) "l'expérience prouva que bien souvent nos prédécesseurs eurent raison d'accélérer ainsi (par le reboisement) les processus naturels, grâce à un important investissement de départ en matériel végétal diversifié et en main-d'œuvre ».

\section{CONCLUSIONS}

Au travers de ces écrits, il nous parvient l'écho d'une démarche volontaire et visionnaire, répondant dans la pratique au besoin nouveau, pour l'époque, de "recréer la nature", pour limiter l'érosion. Force est de constater que les concepts techniques développés, nés des réalisations sur le terrain et validés par celles-ci entre 1865 et 1880 , étaient inédits. D'un point de vue écologique, ils reposent sur des hypothèses en grande partie confirmées par les connaissances postérieures. Pour n'en citer que quelques-unes :

- la part importante de l'action humaine sur la dégradation de la végétation (Beaulieu, 1977 ; Barbéro et al., 1990 ; Thinon, 1992 ; Reille et al., 1996), qui a longtemps été débattue (voir "La légende du déboisement des Alpes" de Lenoble, 1921);

- le rôle primordial de la végétation dans le contrôle de l'érosion et la régulation des eaux dans les petits bassins versants de montagne, notamment sur les marnes noires (Mathys et al., 1996) ;

- la faisabilité écologique et technique de la restauration, y compris sur les marnes noires en érosion, vérifiée par les forêts créées, mais aussi par le développement d'expérimentations nouvelles (Crosaz, 1995) ;

- la définition de l'écosystème réhabilité puis de l'écosystème objectif, que l'on peut envisager en comparaison avec les références avant perturbation humaine forte et par l'étude de la remontée biologique actuelle (Vallauri, 1997a); 


\section{VALLAURI}

- la définition des zones cible pour la restauration, en mettant au premier plan les zones de marnes noires. L'approche gagnerait à l'avenir en précision par une approche en terme d'écologie du paysage (système géomorphologique, types de stations), et en efficacité opérationnelle en mobilisant l'information par un système d'information géographique ;

- la complémentarité de la dynamique naturelle (mise en défens ou déprise) et ses limites pour restaurer les zones dégradées. L'analyse diachronique de certains paysages non restaurés montre par exemple clairement le retard de colonisation des marnes noires, bien que la dynamique n'y soit pas nulle (Vallauri, 1997a) ;

- le choix des espèces pour la réhabilitation, notamment d'espèces forestières pionnières comme le Pin noir (Vallauri, 1997b).

Avec un siècle de recul, ces réalisations et ces techniques demeurent une somme d'expériences insuffisamment valorisées, notamment hors du monde forestier. Cela ne serait pourtant pas sans intérêts dans le cadre des réflexions contemporaines sur la restauration d'écosystèmes ou d'habitats dégradés (terres érodées, zones humides, berges et talus, carrières,...). Quelles techniques de réhabilitation ont fait leurs preuves? Quels critères utiliser pour juger de la réussite de la restauration? Comment évaluer sa viabilité à long terme et, si nécessaire, l'améliorer en adaptant les techniques dès la phase de réhabilitation?

D'autre part, la revue de cette littérature technique met également en perspective les problèmes de renouvellement posés, à l'heure actuelle, dans ces forêts créées. Certes, les auteurs anciens s'attachent, à juste titre, à développer en priorité les techniques de la phase de réhabilitation, dont ils ont un besoin urgent (Mathieu, 1865 ; Demontzey, 1878). Certes, les capacités dynamiques sur le long terme des végétaux installés, et de l'écosystème recréé, leur étaient en partie inconnues. Toutefois, les objectifs de la restauration sont déjà évoqués sur le long terme, aucun de ces deux auteurs n'ayant la prétention de recréer une forêt sur marnes érodées en une étape et en quelques années seulement. Les éclaircies, la diversification des essences, le rôle possible du Chêne pubescent, du Hêtre ou du Sapin dans ces forêts nouvelles, réflexions très actuelles de la phase d'accompagnement, sont déjà développés par Lavauden en 1933. Elles ne seront malheureusement suivies que de peu de travaux. Ceux-ci débutèrent pour répondre à l'urgence au cours de la décennie 1980, sous l'impulsion de Combes, Bartet et de Crécy notamment. Leur réalisation pose aujourd'hui encore des problèmes écologiques et techniques sur les stations les plus sèches (marnes noires par exemple).

Quant à l'épilogue, la gestion durable de ces forêts de protection, tout reste à discuter, à expérimenter d'un point de vue écologique et sylvicole, et surtout à développer sur le terrain, dans un domaine pourtant clé de la foresterie de protection. Quelle sylviculture est adaptée pour la maîtrise durable de l'érosion? Quelle gestion forestière minimale est possible? Une gestion conservatoire seule peut-elle être envisageable? Quel degré d'insertion dans le développement local est nécessaire pour compenser les surcoûts de gestion? Enfin, comment garder la mémoire de la sensibilité des milieux à l'érosion?

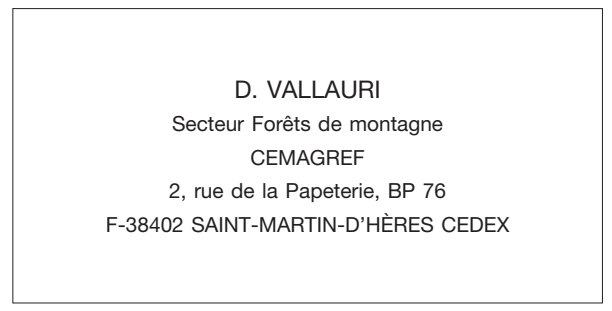




\section{BIBLIOGRAPHIE}

BARBERO (M.), BONIN (G.), LOISEL (R.), QUEZEL (P.). - Changes and disturbances of forest ecosystems caused by human activities in the western part of mediterranean basin. - Vegetatio, vol. 87, 1990, pp. 151-173.

BEAULIEU (J.-L. de). - Contribution pollen analytique à l'histoire tardiglaciaire et holocène de la végétation des Alpes méridionales françaises. - Université Aix-Marseille III, 1977. - 358 p. (Thèse ès-Sciences).

COMBES (F.), BARTET (J.-H.). - Plaidoyer pour le Pin noir en Haute-Provence. - Revue forestière française, vol. XXXIV, $n^{\circ} 5$ spécial "Restauration des Terrains en Montagne", 1982, pp. 40-49.

CRÉCY (L. de). - La Restauration des terrains en montagne, actualité d'une entreprise centenaire. - Revue forestière française, vol. XXXIV, n 5 spécial "Restauration des Terrains en Montagne", 1982, pp. 7-17.

CROSAZ (Y.). - Lutte contre l'érosion des sols en montagne méditerranéenne. Connaissance du matériel végétal et quantification de son impact sur l'érosion. - Université d'Aix-Marseille III, 1995. - 204 p. + annexes (Thèse de $3^{\mathrm{e}}$ cycle).

DEMONTZEY (P.). - Étude sur les travaux de reboisement et de gazonnement des montagnes. - Paris : Imprimerie nationale, 1878 (nouvelle édition publiée en 1882). - $421 \mathrm{p}$.

DOUGUEDROIT (A.). - Les Périmètres de reboisement dans les Alpes du Sud. - Revue forestière française, vol. XXXII, $\mathrm{n}^{\circ}$ spécial "Société et forêts", 1980, pp. 37-45.

DUGIED (P.H.). - Projet de boisement des Basses Alpes. - Paris : Roy, 1819. - 113 p.

FABRE (citoyen). - Essai sur la théorie des torrents et des rivières. $-1797 .-482 p$.

LAVAUDEN (L.). - Instruction sommaire sur les reboisements dans les Basses-Alpes. - Digne, 1933. - 7 p.

LENOBLE (F.). - La Légende du déboisement des Alpes. - Revue de Géographie alpine, tome XI, 1923, pp. 5-116.

MATHIEU (M.A.). - Le Reboisement et le regazonnement des Alpes. - Paris, 1865. - 112 p.

MATHYS (N.), BROCHOT (S.), MEUNIER (M.). - L'Érosion des Terres Noires dans les Alpes du Sud: contribution à l'estimation des valeurs annuelles moyennes (bassins versants expérimentaux de Draix, Alpes-de-Haute-Provence, France). - Revue de Géographie alpine, ${ }^{\circ} 2$ 2, 1996, pp. 17-27.

PONCET (A.). - Restauration et conservation des terrains en montagne. - ONF, 1994. - 1000 p.

RAUCH (F.A.). - Régénération de la nature végétale, ou recherches sur les moyens de recréer, dans tous les climats, les anciennes températures et l'ordre primitif des saisons, par des plantations raisonnées, appuyées de quelques vues sur le ministère que la puissance végétale semble avoir à remplir dans l'harmonie des éléments. - Paris: Didot L'Ainé, Imprimeur du Roi, 1818. - 502 p.

REILLE (M.), ANDRIEU (V.), BEAULIEU (J.-L. de). - Les grands traits de l'histoire de la végétation des montagnes méditerranéennes occidentales. - Écologie, tome 27, n 3, 1996, pp. 153-169.

SURREL (A.). - Étude sur les torrents des Hautes-Alpes. - Paris, 1841. - 284 p.

THINON (M.). - L'Analyse pédoanthracologique. Aspects méthodologiques et applications. - Université de Marseille Saint-Jérôme, 1992. - 317 p. (Thèse d'État).

VALLAURI (D.). - Dynamique de la restauration forestière des substrats marneux avec Pinus nigra J.F. Arnold ssp. nigra dans le secteur haut-provençal. Trajectoires dynamiques, avancement du processus de restauration et diagnostic sur l'intégrité fonctionnelle des écosystèmes recréés. - Université de Marseille III, Cemagref de Grenoble, 1997a. 300 p. + annexes (Thèse de Doctorat).

VALLAURI (D.). - Diversité végétale : un atout pour la restauration des espaces forestiers dégradés. Le cas de la RTM sur substrats marneux dans les Préalpes du Sud. - Forêt méditerranéenne, tome XVIII, n 1, 1997b, pp. 26-29.

VALLAURI (D.), CHAUVIN (Ch.). - Les Concepts de l'écologie de la restauration appliqués à la forêt. - Revue forestière française, vol. XLIX, n 3, 1997, pp. 195-203.

VALLAURI (D.), CHAUVIN (Ch.), MERMIN (E.). - La Restauration écologique des espaces forestiers dégradés dans les Alpes du Sud. Chronique de 130 ans de restauration et problématique actuelle des forêts recréées en Pin noir. Revue forestière française, vol. XLIX, $\mathrm{n}^{\circ} 5,1997, \mathrm{pp} .433-449$.

\section{RELECTURE PAR UN ÉCOLOGUE DES PRINCIPAUX ÉCRITS SUR LA RESTAURATION D’ESPACES ÉRODÉS DANS LES ALPES DU SUD (1797-1994) (Résumé)}

La restauration d'espaces dégradés par érosion est une entreprise ambitieuse mais indispensable pour remettre les territoires dégradés sur le chemin du développement durable. La politique de Restauration des Terrains en Montagne (RTM) dans les Alpes du Sud en offre un exemple ancien et inestimable, tant sur le plan écologique et technique que politique. À partir d'extraits d'écrits sur les techniques de la RTM, de ses précurseurs à ses acteurs contemporains, les évolutions des idées sur la restauration et les logiques d'un siècle d'actions forestières sont soulignées. Les actions passées et celles nécessaires actuellement sont remises en perspectives dans le contexte écologique du programme. 


\section{VALLAURI}

THE MAJOR DOCUMENTS OF THE RESTORATION OF BADLANDS IN THE SOUTHERN ALPS (1797-1994) REVISITED BY AN ECOLOGIST (Abstract)

Restoration of badlands is an ambitious but inescapable undertaking to redirect eroded landscapes towards sustainable development. The French forest service for erosion control in the south-western Alps offers a long-established and invaluable example in all respects - ecological, technical and political. A survey of the principles of restoration and the rationale followed over a century of forestry interventions is provided through a review of the service's technical literature from the pioneers in the XIXth century to current leaders. Past operations and currently required actions are set in the perspective of the program's ecological framework. 